\title{
Increased plasma concentration of epidermal growth factor in female patients with non-toxic nodular goitre
}

\author{
Jan Brzeziński ${ }^{1}$ and Andrzej Lewiński ${ }^{2}$ \\ ${ }^{1}$ Department of Endocrine Surgery and ${ }^{2}$ Department of Thyroidology, Institute of Endocrinology, Medical University of Łódź, Poland \\ (Correspondence should be addressed to A Lewiński, Department of Thyroidology, Institute of Endocrinology, 5, Dr. Sterling St, 91-425 Łódź, Poland)
}

\begin{abstract}
Objective: Epidermal growth factor (EGF) is believed to be one of the most potent growth factors for the thyroid gland.

Design and methods: We tested plasma EGF concentrations (radioreceptor method) in patients with nontoxic nodular goitre (NNG). Blood samples from patients with NNG were collected on the day before the surgery, during subtotal thyroidectomy (sTx) - from peripheral and thyroid veins, on the 3rd day after sTx, 3 months after sTx, and 9 months after the surgery.

Results: Before the operation, the mean plasmaEGF concentration in 50 women with NNG was significantly higher $(1.16 \pm 0.69 \mathrm{ng} / \mathrm{ml}$; mean \pm s.e. $)$ than that in 32 control female patients subjected subsequently to other (non-thyroid) operations $(0.41 \pm 0.27 \mathrm{ng} / \mathrm{ml} ; P<0.001)$, and that in 20 healthy women $(0.35 \pm 0.11 \mathrm{ng} / \mathrm{ml} ; P<0.001)$, both of which groups were without goitre or had a small non-toxic diffuse goitre. The surgical treatment of NNG resulted in a significant reduction in plasma EGF 3 months after sTx $(0.56 \pm 0.22 \mathrm{ng} / \mathrm{ml})$. A further slight decrease was observed 9 months after sTx $(0.44 \pm 0.14 \mathrm{ng} / \mathrm{ml})$.

Conclusions: Our observations confirm the assumption that EGF may participate in the process of goitre formation in human subjects. However, the source of EGF involved in this process remains unclear. Some findings could suggest that plasma EGF, which was increased in patients with NNG, may be of non-thyroid origin; however, the majority of our observations strongly stress the significance of an intact thyroid in the preservation of an unchanged EGF value, suggesting a role of locally produced EGF in the pathogenesis of NNG.
\end{abstract}

European Journal of Endocrinology 138 388-393

\section{Introduction}

The involvement of tissue growth factors in the process of goitre formation is a problem of a great significance in clinical medicine, as numerous theories on the aetiopathogenesis of the thyroid enlargement or cancer assign an essential role to, or are even dependent upon, the action of the growth factors.

The demonstration of epidermal growth factor (EGF) receptors on the membranes of porcine and human thyrocytes was one of the first indications to suggest its participation in the regulation of the thyroid growth and secretion (1). The presence of immunoreactive EGF has been reported in both human (2) and murine thyroid (3), raising the question of whether thyroidal EGF is, in reality, produced in the thyroid gland.

It has been reported that thyroxine $\left(\mathrm{T}_{4}\right)$ decreased plasma EGF concentration, but increased the thyroid EGF concentration in adult male BALB/c mice (4). Consistent with this, it has also been demonstrated that $\mathrm{T}_{4}$ increased the levels of EGF mRNA in the thyroids of male BALB/c mice (5), thus implying a possibility of synthesis of this growth factor in the thyroid. In contrast, it is known that EGF inhibits the synthesis of thyroid hormones and their peripheral metabolism; a marked decrease in circulating concentrations of triiodothyronine $\left(\mathrm{T}_{3}\right)$ and $\mathrm{T}_{4}$ in sheep was noted after an infusion of EGF (6).

There is a prevalent opinion that EGF in vitro stimulates the proliferation of thyroid follicular cells (TFC) (7). One significant observation was that TSH potentiated EGF binding with its receptors on thyrocytes and also enhanced the mitogenic effect of EGF on the thyroid (8). Other authors have failed to observe a proliferogenic effect of EGF on thyrocytes of rat strain FRTL in cell culture, possibly because of a lack of EGF receptors in the FRTL-5 cells (9). However, opposing reports also exist: both the presence of EGF receptors (10) and the EGF-induced stimulation of cell proliferation (11) have recently been reported for the FRTL-5 cell line.

Apart from the thyroid cell cultures and suspension cultures of thyroid follicles, EGF has been demonstrated to stimulate TFC growth in organ-cultured rat thyroid 
lobes (12) and in in vivo conditions, after transplantations of thyroid tissue into nude mice (newborn rat thyroids (13); differentiated human thyroid cancer cell lines (14) and human Graves' disease tissue (15)).

The goal of the present study was to measure plasma EGF concentrations and serum thyroid-stimulating hormone ( $\mathrm{TSH}), \mathrm{T}_{4}$ and $\mathrm{T}_{3}$ concentrations in patients with non-toxic nodular goitre (NNG) before subtotal thyroidectomy (sTx), during the surgery and at three different time points after the surgery (3 days, 3 months, 9 months). Patients with no goitre or with a small non-toxic diffuse goitre served as controls.

\section{Subjects and methods}

Fifty female patients with NNG (aged $46.8 \pm 10.0$ years; mean \pm s.e.), treated surgically at the Department of Endocrine Surgery, Medical University of Łódź, and two groups ( $\mathrm{A}$ and $\mathrm{B})$ of female controls were included in the study. Control group A consisted of 20 healthy women aged $36.3 \pm 8.6$ years and control group B were 32 women aged $49.5 \pm 9.9$ years who underwent other (non-thyroid) operations (26 - cholecystectomy for chronic calculous cholecystitis and six - appendectomy because of appendicitis).

All the thyroid operations were performed by a team of surgeons directed by one of us ( J B). No complications were observed after the surgery. In the postoperative period, 7 days after thyroid surgery, when histopathological confirmation of the preoperative cytological diagnosis became available and revealed a benign nature of the thyroid lesions, the patients began receiving $\mathrm{L}_{-} \mathrm{T}_{4}(100 \mu \mathrm{g} /$ daily $)$ and continued that treatment for a period exceeding the duration of the present study.

The size of the thyroid gland was determined, by palpation, according to the WHO/UNICEF/ICCIDD classification of goitre (16) and was confirmed by ultrasound examination of the thyroid gland volume. Among control group A, no goitre was found in 18 (grade 0 or $1 \mathrm{~A}$ in the above classification) and a small non-toxic diffuse goitre (grade 1B) was observed in two; the respective numbers among control group $B$ were 30 and two.

All the patients subjected to sTx had an NNG of grade 2 or 3 (19 and 31 patients respectively). After the surgery, the removed thyroid mass was measured in all the patients.

Blood samples from the patients with NNG were collected at five time points: on the day before surgery (time point No. 1); during sTx (when it was drawn both from the peripheral and from the thyroid veins; time point No. 2); on the 3rd day after sTx (time point No. 3); 3 months after the surgery (time point No. 4) and 9 months after the operation (time point No. 5). Blood samples from control group B, were collected at two time points: on the day before the non-thyroid surgery (time point No. 1) and 3 months after the surgery (time point No.4).
Plasma EGF concentrations were tested by a radioreceptor method, using EGF Radioassay Kits (Biomedical Technologies Inc., Stoughton, MA, USA). In order to avoid coagulation, blood samples were collected into high-quality borosilicate glass tubes, with addition of EDTA as an anticoagulant $(7.2 \mathrm{mg} / 5 \mathrm{ml}$ of whole blood). To prevent proteolysis, Trasylol was added $(500 \mathrm{KIU} / \mathrm{ml})$ and the tubes were placed on ice before centrifugation (15 min at 2200 r.p.m.), also under refrigeration. After that, the plasma samples were collected in plastic tubes and kept in a freezer at $-25^{\circ} \mathrm{C}$ until required for assay. The lower limit of EGF detection was $0.20 \mathrm{ng} / \mathrm{ml}$. The percentage of recovery after the immediate addition of exogenous EGF to the blood sample was $97 \%$; after deep-freeze storage for 2 weeks and a subsequent defrosting (i.e. during the radioreceptor assay) it was $88 \%$. The intra-assay coefficient of variation was $8.4 \%$.

Serum $\mathrm{T}_{3}, \mathrm{~T}_{4}$ and TSH concentrations were determined in all the patients with NNG and in both control groups at each of the time points, by fluoroimmunoassay, with the use of reagents manufactured by Wallac Oy, Turku, Finland (DELFIA kits).

It should be noted that patients with any essential metabolic disorders, particularly with diseases affecting plasma protein concentration, were not included in the study. The remaining patients in the NNG group were included consecutively in the experiment, and then operated on. The final criterion for inclusion of the patients into the NNG group was a result from postoperative histopathological examination of excised thyroid tissue that confirmed the diagnosis of nodular goitre.

\section{Statistical analyses}

Evaluation of the EGF concentrations was performed using a test for dependent variates, based on $T^{2}$-Hotelling's statistics (17). This test is appropriate to the assessment of repeated measures of parameters, for example those performed on the same objects at sequential time points. The statistical differences between the data were analysed for successive pairs of time points.

Analysis of the profile of data for EGF concentrations was used for two independent groups (NNG group and control group B), on the basis of $T^{2}$-Hotelling's statistics (17). This test may be applied to demonstrate interactions between particular groups and respective results. Two time points were considered: No. 1, the day before the surgery, and No. 4, 3 months after the operation. Keeping the same purpose in mind, a twofactorial ANOVA [where factor $\mathrm{A}=$ time (before the surgery or 3 months after the surgery) and factor $\mathrm{B}=$ group (patients with NNG, control group A, control group B)] was performed.

Correlation coefficients $(r)$ were calculated for the analysis of interdependence between the concentrations of particular hormones and EGF. 
Table 1 Plasma concentrations of EGF and serum concentrations of $\mathrm{TSH}, \mathrm{T}_{4}$ and $\mathrm{T}_{3}$ in the groups studied, at various time points of blood collection. Values are mean \pm S.E.

\begin{tabular}{lcccc}
\hline Time point of blood collection & $\begin{array}{c}\text { EGF } \\
(\mathrm{ng} / \mathrm{ml})\end{array}$ & $\begin{array}{c}\text { TSH } \\
(\mathrm{mlU} / \mathrm{l})\end{array}$ & $\begin{array}{c}\mathbf{T}_{4} \\
(\mathrm{nmol} / \mathrm{l})\end{array}$ & $\begin{array}{c}\mathbf{T}_{3} \\
(\mathrm{nmol} / \mathrm{l})\end{array}$ \\
\hline Normal range & & $0.3-4.0$ & $69-141$ & $1.3-2.5$ \\
Patients with NNG & & & & \\
$\quad$ 1: Before sTx (day before sTx) & $1.16 \pm 0.69$ & $1.1 \pm 0.6$ & $113.7 \pm 21.8$ & $1.6 \pm 0.2$ \\
2: During sTX & & & $107.4 \pm 17.6$ & $1.6 \pm 0.3$ \\
$\quad \quad$ Peripheral vein & $0.95 \pm 0.62$ & $1.2 \pm 0.7$ & $115.5 \pm 18.1$ & $1.8 \pm 0.4$ \\
$\quad \quad$ Thyroid vein & $0.73 \pm 0.51$ & $1.3 \pm 0.7$ & $96.1 \pm 17.8$ & $1.0 \pm 0.3$ \\
3. 3 days after sTx & $0.88 \pm 0.50$ & $1.6 \pm 0.8$ & $118.7 \pm 18.1$ & $1.6 \pm 0.4$ \\
4. 3 months after sTx & $0.56 \pm 0.22$ & $3.4 \pm 2.8$ & $124.0 \pm 14.6$ & $1.6 \pm 0.3$ \\
5: months after STx & $0.44 \pm 0.14$ & $3.3 \pm 2.3$ & $100.1 \pm 17.1$ & $1.5 \pm 0.2$ \\
Control group A & $0.35 \pm 0.11$ & $1.2 \pm 0.4$ & & \\
Control group B & & & $102.9 \pm 17.9$ & $1.5 \pm 0.5$ \\
$\quad$ 1: Day before non-thyroid surgery & $0.41 \pm 0.27$ & $1.2 \pm 0.6$ & $106.9 \pm 15.7$ & $1.4 \pm 0.4$ \\
$\quad$ 4: 3 months after non-thyroid surgery & $0.34 \pm 0.11$ & $1.1 \pm 0.6$ & \\
\hline
\end{tabular}

sTx, subtotal thyroidectomy. Control group A, healthy women without goitre (18) or with a small non-toxic diffuse goitre (2). Control group B, patients subjected to non-thyroid surgery, without goitre (30) or with a small non-toxic diffuse goitre (2).

Student's $t$-test or Satterthwaite's test was used for comparison of the concentrations of EGF or of examined hormones in the thyroid blood and peripheral blood, preceded by comparison of variances with the $\mathrm{F}$ test, based on the Snedecor's distribution.

\section{Results}

Numerical data concerning plasma concentrations of EGF and serum concentrations of TSH, $\mathrm{T}_{4}$ and $\mathrm{T}_{3}$ are presented in Table 1. Concentrations of EGF in blood at the five time points of collection are illustrated in
Fig. 1, together with profile data analysis for two groups (the NNG group and control group B) and for two time points (Nos 1 and 4).

The changes in mean plasma EGF concentration over time were statistically significant $(P<0.005)$. The timedependence of EGF concentration in the NNG group was revealed as a tendency towards a gradual decrease in the concentration in question, and was apparent as early as 3 days after sTx and became much more pronounced 3 months and 9 months after the surgery. The differences between the successive time points were statistically significant $(P<0.001)$, except for the time

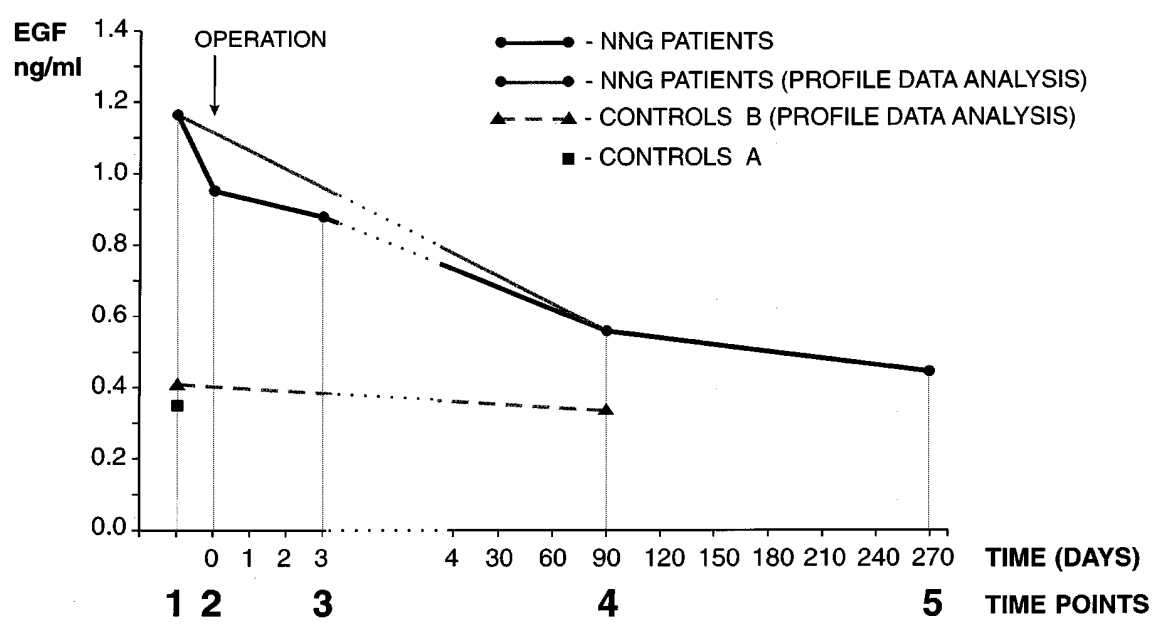

Figure 1 Mean plasma EGF concentrations in the NNG group at the various time points of blood collection and profile analysis of EGF concentrations in the NNG group (continuous grey line) and control group B (dashed grey line) at time points 1 and 4 . For details of time points, see Subjects and methods. Time point No. 2 (the day of operation) is shown on the figure as day ' 0 '. Controls A were non-operated healthy patients without goitre or with a goitre of small size; controls B were patients without goitre or with a goitre of a small size who were treated surgically for non-thyroid diseases; NNG patients had non-toxic nodular goitre. There is a time-dependence of the EGF concentration $(P<0.05)$. Significant differences between subsequent pairs of time points are: 1 vs $2, P<0.001 ; 2$ vs $3, P>0.05 ; 3$ vs 4 , $P<0.001 ; 4$ vs $5, P<0.001$. There is a significant decrease in EGF concentration in the NNG group $(P<0.001)$, but no such significant difference in control group $\mathrm{B}(P>0.05)$. 
points Nos 2 and 3, for which the difference did not attain statistical significance $(P>0.05)$ (Fig. 1$)$.

The profile data of EGF concentrations were significantly different between control group B and the NNG group $(P<0.001)$. When the mean EGF concentration in control group B before surgery was compared with that 3 months after the operation, no significant difference was observed $(P>0.05)$, whereas a significant decrease of EGF concentration occurred in the NNG group over the same period ( $P<0.001$; Fig. 1$)$.

EGF concentrations in control group $A$ and control group B (at time point No. 1) did not differ significantly statistically, but at the same time point, the EGF concentration was significantly greater in the patients with NNG than in control groups A or B.

At time point No. 4 of blood collection (3 months after the surgery), the plasmaEGF concentration was greater in the patients after sTx, compared with that in control group B (after operation for non-thyroid disease).

A similar statistical analysis of changes in TSH concentrations (data not shown) produced the following results: (i) TSH concentrations did not differ significantly in control groups $\mathrm{A}$ and $\mathrm{B}$ and in the patients with NNG before surgery: (ii) TSH concentrations began to increase on the 3rd day after sTx, the increase being most pronounced 3 months after surgery, after which some plateauing was observed: (iii) the concentration in sTx patients, 3 months after surgery, was significantly greater than that in control group $\mathrm{B}$ at the same time point $(P<0.001)$ : (iv) in contrast with the findings in the NNG group, no timedependence of TSH concentrations was noted for control group B.

Correlation coefficients $(r)$ demonstrated a negative correlation between the concentrations of EGF and

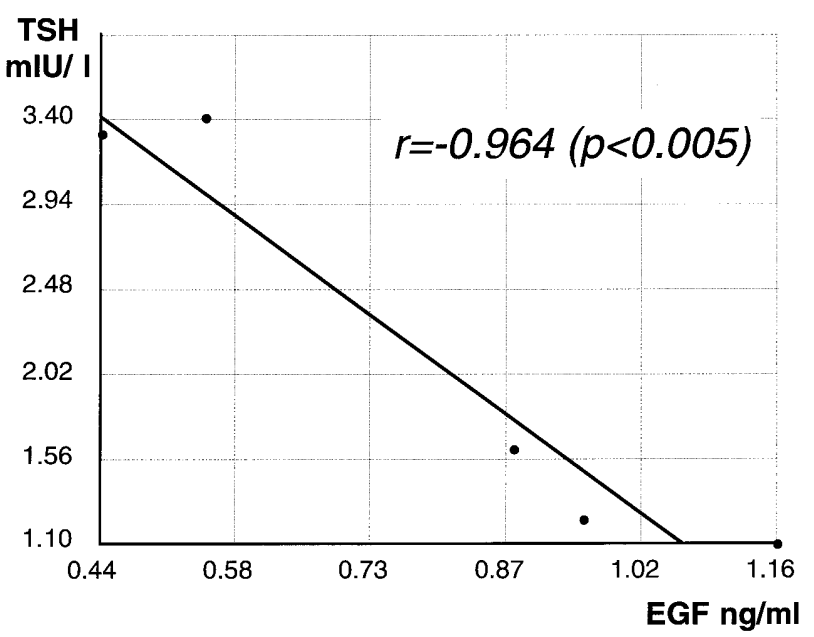

Figure 2 Negative correlation between the concentrations of EGF and TSH in patients with non-toxic nodular goitre in the course of the study. $r$, correlation coefficient.
TSH (Fig. 2); no correlations between EGF concentration and $\mathrm{T}_{4}$ or $\mathrm{T}_{3}$ concentrations $(r=-0.58$ and $r=0.04$ respectively; data not shown); and a strong positive correlation (as expected) between the mass (measured postoperatively) and the volume (examined before surgery) of the gland $(r=0.9601)$ (data not shown).

The mean plasma EGF concentration in blood collected from the peripheral vein was greater than that in the thyroid vein $(P<0.001)$.

There was no correlation between the platelet counts and plasma EGF concentrations in the NNG group, the correlation coefficient value being negative and statistically not significant $(r=-0.551 ; P=0.34)$.

\section{Discussion}

The present data show clearly that patients with NNG have greater plasma concentrations of EGF than do controls without goitre or with a small non-toxic diffuse goitre. Moreover, in the NNG group there were correlations between the thyroid volume, as examined before sTx, or between the mass of the removed gland, and the preoperative EGF concentration. No correlations of thyroid volume with plasma EGF concentrations were found in either control group.

Our observations are consistent with the widely held view that EGF is a potent growth factor for the thyroid and suggest that EGF may participate in the mechanism of formation of nodular goitre in humans.

In the present study, control group B patients were treated surgically because of chronic cholecystitis or appendicitis. The plasma EGF concentration in that group did not differ from the concentrations detected in healthy women (control group A), and did not change in a statistically significant manner, after the surgical treatment of the non-thyroid disease. It should be stressed that no evidence has been published to suggest that plasma EGF concentrations are altered in inflammatory diseases. It is also important to note that both the demonstration of a time-dependence of changes in EGF concentration, and the analysis of profile data indicate clearly that, in contrast to findings in control group B, the concentration of EGF in patients with NNG decreased after sTx.

It is well known that, in human subjects, serum EGF is mainly derived from platelets (18) and that the difference between the serum and plasma EGF concentrations results from the release of platelet EGF into serum during the process of clotting. The origin of plasma EGF remains unclear, although the possibility exists of a leakage of the platelet EGF content into plasma. In the present study, blood samples were protected adequately against coagulation and proteolysis, and the platelet counts in all the patients examined patients were within normal ranges. There was no correlation between the platelet counts and plasma EGF concentrations in the NNG group. 
Despite the relationship between the increased EGF concentration and the presence of NNG, some of our findings could suggest that plasma EGF, which was increased in patients with nodular goitre, may be of non-thyroid origin. This conclusion can be drawn on the basis of our observation that plasma EGF concentration did not decrease instantaneously after the surgery, but reduced gradually, accompanying, at least at the beginning of the postoperative period, the decrease in $T_{3}$ and $T_{4}$ concentrations. Moreover, in our present study, the concentration of EGF in blood collected from the thyroid vein was less than that in the peripheral blood.

The majority of our observations, however, emphasize the significance of an intact thyroid in the preservation of unchanged EGF concentrations, keeping open the problem of the source of plasma EGF. The relevant observations are the following: the existence of correlations between the thyroid volume or the mass of the gland and the preoperative EGF concentration, as demonstrated in the NNG group; a lack of restoration of EGF concentrations to those found before sTx, in spite of administration of $\mathrm{L}_{-} \mathrm{T}_{4}$ and a full recovery of the patients to the euthyroid state; the presence of increased plasma concentrations of EGF in patients with NNG before sTx, with no evidence for the development of hyperplastic lesions in other epithelial tissues or organs. These observations strongly suggest a local effect of EGF in the thyroid. The recent detection of EGF mRNA in thyroid extracts (5) provides definitive proof of EGF synthesis within the gland; however, direct evidence for hyperplastic or hypertrophic action of the locally produced EGF is still lacking.

In the present investigation, we observed a negative correlation between EGF and TSH concentrations, based on the calculation of results from five time points of blood collection. No correlations were detected between EGF and $\mathrm{T}_{4}$ concentrations, or between EGF and $\mathrm{T}_{3}$ concentrations, which may have been the result of postoperative administration of $\mathrm{L}_{\mathrm{T}} \mathrm{T}_{4}$ to the patients with NNG.

The reciprocal relationships between EGF and thyroid hormone concentrations are difficult to interpret with clarity. An excess of endogenous $\mathrm{T}_{4}$, as in the case of thyrotoxic patients, has been shown to increase serum and plasma EGF concentrations (19). In contrast, deficiency of $\mathrm{L}-\mathrm{T}_{4}$ may result in a decrease in EGF concentration; it has consistently been reported that hypothyroid subjects had unmeasurable plasma EGF (19).

In contrast to the stimulatory action of EGF on thyroid growth processes in vivo and in vitro (resulting in dedifferentiation of TFC), EGF has repeatedly been shown to inhibit thyroid function $(6,7,13)$. Thus the negative effect of EGF on thyroid hormone production may represent a feedback control mechanism, balancing the induction of EGF secretion caused by thyroid hormones.
In conclusion, our results suggest that EGF may participate in the mechanism of development of nodular goitre in humans. However, some of our observations speak in favour of the hypothesis that plasma EGF, increased in patients with NNG, may be of non-thyroid origin. Nevertheless, the correlations between the preoperative EGF concentration and the thyroid volume or the mass of the gland, recorded in patients with NNG, the failure of $\mathrm{L}-\mathrm{T}_{4}$ administration to reverse the decrease in EGF concentration in patients after sTx and, finally, the occurrence of growth reactions only in the thyroid glands of patients with NNG and not in other tissues and organs, all serve to contradict the above concept, strongly indicating the essential role of an intact thyroid as the source of EGF.

\section{References}

1 Kasai K, Kuroda H, Hashigami Y, Ishikawa M, Nakamura T \& Shimoda SI. Specific epidermal growth factor receptors on porcine and human thyroid membranes. Hormone and Metabolic Research $198517592-594$.

2 Hirata Y \& Orth DN. Epidermal growth factor (urogastrone) in human tissues. Journal of Clinical Endocrinology and Metabolism $197948667-672$.

3 Dagogo-Jack S, Atkinson S \& Kendall-Taylor P. Demonstration of significant epidermal growth factor activity in murine thyroid tissue. Journal of Endocrinology $198611067-72$.

4 Ozawa S, Sheflin LG \& Spaulding SW. Thyroxine increases epidermal growth factor levels in the mouse thyroid in vivo. Endocrinology 1991128 1396-1403.

5 Sheflin LG, Fucile NW, Ozawa S \& Spaulding SW. Thyroxine increases the levels of epidermal growth factor messenger ribonucleic acid (EGF mRNA) in the thyroid in vivo, as revealed by quantitative reverse transcription polymerase chain reaction with an internal control EGF mRNA. Endocrinology 1993132 2319-2324.

6 Corcoran JM, Waters MJ, Eastman CJ \& Jorgensen G. Epidermal growth factor: effect on circulating thyroid hormone levels in sheep. Endocrinology $1986119214-217$.

7 Westermark K \& Westermark B. Mitogenic effect of epidermal growth factor on sheep thyroid follicle cells in culture. Experimental Cell Research 1982138 47-55.

8 Westermark K, Karlsson FA \& Westermark B. Thyrotropin modulates EGF receptor function in porcine thyroid follicle cells. Molecular and Cellular Endocrinology 198540 17-23.

9 Pang X-P \& Hershman JM. Differential effects of growth factors on $\left[{ }^{3} \mathrm{H}\right]$ thymidine incorporation and $\left[{ }^{125} \mathrm{I}\right]$ iodine uptake in FRTL-5 rat thyroid cells. Proceedings of the Society for Experimental Biology and Medicine 1990194 240-244.

10 Sugawa H, Beniko M, Imura H \& Mori T. Characterization of epidermal growth factor receptor in a rat thyroid cell line, FRTL-5. Biochemical and Biophysical Research Communications 1993193 390-397.

11 Asmis LM, Gerber H, Kaempf J \& Studer H. Epidermal growth factor stimulates cell proliferation and inhibits iodide uptake of FRTL-5 cells in vitro. Journal of Endocrinology 1995145 513-520.

12 Żerek-Mełeń G, Lewiński A \& Szkudliński M. Influence of somatostatin and epidermal growth factor (EGF) on the proliferation of thyroid follicular cells in organ culture. Regulatory Peptides 199028 293-300.

13 Ozawa S \& Spaulding SW. Epidermal growth factor inhibits radioiodine uptake but stimulates deoxyribonucleic acid synthesis in newborn rat thyroids grown in nude mice. Endocrinology 1990 $127604-612$.

14 Hölting T, Siperstein AE, Clark OH \& Duh QY. Epidermal growth factor enhances proliferation, migration, and invasion of 
follicular and papillary thyroid cancer in vitro and in vivo. Journal of Clinical Endocrinology and Metabolism 199479 401-408.

15 Paschke R, Erk T, Herfurth J \& Usadel KH. Stimulation of proliferation and inhibition of function of xenotransplanted human thyroid tissue by epidermal growth factor. Journal of Endocrinological Investigation 199518 359-363.

16 Delange F, Bastani S, Benmiloud M, De Maeyer E, Isayama MG, Koutras D et al. Definitions of endemic goiter and cretinism, classification of goiter size and severity of endemias, and survey techniques. In Towards the Eradication of Endemic Goiter, Cretinism and Iodine Deficiency, vol 5, pp. 373-376. Eds JT Dunn, E Pretell, CH Daza \& FE Viteri. Washington: PAHO Sc. Publ., 1986.

17 Morrison DF. Multivariate Statistical Methods [in Polish]. Warszawa: Pañstwowe Wydawnictwo Naukowe, 1990.
18 Pesonen K, Viinikka L, Myllyla G, Kiura J \& Perheentupa J. Characterisation of material with epidermal growth factor immunoreactivity in human serum and platelets. Journal of Clinical Endocrinology and Metabolism 198968 486-491.

19 Kung AWC, Hui WM \& Ng ESK. Serum and plasma epidermal growth factor in thyroid disorders. Acta Endocrinologica $199212752-57$.

Received 10 December 1997

Accepted 22 December 1997 